\title{
Quantum dot multi-wavelength lasers for Tbit/s coherent communications and 5G wireless networks -INVITED
}

\author{
Z.G. Lu, J.R. Liu, Y.X. Mao, K. Zeb, G.C. Liu, J. Webber, M. Rahim, G. Pakulski, \\ C.Y. Song, M. Vachon, P. Barrios, D. Poitras, S.R. Wang, W.H. Jiang, and P. Poole \\ Advanced Electronics and Photonics (AEP) Research Centre, National Research Council (NRC) Canada, Ottawa, ON, Canada
}

\begin{abstract}
We will present the design, growth, fabrication, electronic control and packaging of the InAs/InP quantum dot (QD) multi-wavelength lasers (MWLs) developed by AEP of NRC in Canada. Their key technical specifications include L-I-V curves, optical and RF beating spectra, relative intensity noise (RIN), and optical phase noise of each individual wavelength channel, as well as timing jitter are investigated. Data bandwidth transmission capacity of $5.376 \mathrm{Tbit} / \mathrm{s}$ and $10.3 \mathrm{Tbit} / \mathrm{s}$ respectively in the PAM-4 and 16-QAM modulation formats are demonstrated by only using a single QD MWL chip. We have also developed a novel monolithic InAs/InP QD dual-wavelength (DW) DFB laser as a compact optical beat source to generate millimeter-wave (MMW) signals. Because of using a common cavity, highly coherent and correlated optical modes with optical linewidth as low as $15.83 \mathrm{kHz}$, spectrally pure MMW signals around $46.8 \mathrm{GHz}$ with a linewidth down to 26.1 $\mathrm{kHz}$ were experimentally demonstrated. By using this QD DW-DFB laser, a 1 Gbaud (2Gbps) MMW over-fiber transmission link is demonstrated with PAM-4 signals. The results show that the demonstrated device is suitable for high speed high capacity MMW fiber-wireless integrated fronthaul of $5 \mathrm{G}$ networks.
\end{abstract}

\section{Introduction}

As worldwide data traffic continues to grow, it becomes urgent to utilize communication resources optimally, facilitating capacity growth at reduced energy consumption. Quantum dot (QD) semiconductor multi-wavelength lasers (MWLs) are promising light sources for Terabit/s denseWDM optical coherent networks, optical signal processing and millimeter wave (MMW) generation because of many unique advantages as compared with quantum well (QW) and bulk semiconductor materials [1]. Such monolithic QDbased semiconductor MWLs solve the obvious cost, power consumption and packaging problems by replacing many separate lasers for each channel by only a single semiconductor laser chip. Other advantages include compact size, low power consumption, simple fabrication, and the ability for hybrid integration with silicon substrates. In recent years we have successfully developed InAs/InP QD MWLs with repetition rates from $10 \mathrm{GHz}$ to $437 \mathrm{GHz}$ and a total output power up to $50 \mathrm{~mW}$ per facet at room temperature [1-5]. In this paper we give an overview of the performance of the QD MWLs and their applications in Terabit/s optical communication links and 5G wireless networks.

\section{QD materials and QD MWLs for Tbit/s networks}

The InAs/InP QD material was grown by chemical beam epitaxy (CBE) on exactly (001)-oriented $n$-type InP substrates [6]. A $355 \mathrm{~nm}$ thick InGaAsP waveguide core contained five stacked layers of InAs QDs with $\mathrm{In}_{0.816} \mathrm{Ga}_{0.184} \mathrm{As}_{0.392} \mathrm{P}_{0.608} \quad(1.15 \mathrm{Q})$ barriers as the gain medium, surrounded by $n$ - and $p$ - type InP cladding layers. Single lateral mode ridge waveguide QD-MWLs were fabricated with a stripe width of $2.0 \mu \mathrm{m}$ and a Fabry-Perot (F-P) laser cavity via standard photolithography and combination of dry-, wet-etching and contact metallization techniques. Fig. 1(a) shows a scanning electron microscope (SEM) image and a corresponding schematic cross-section is shown in Fig. 1 (b).

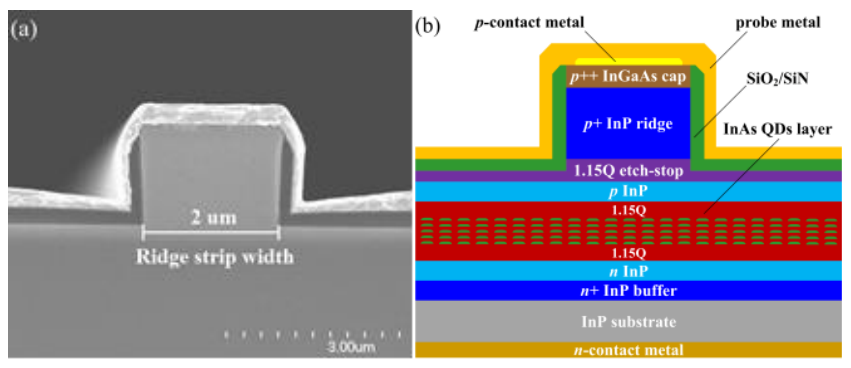

Fig. 1. (a) Cross-sectional SEM image showing the facet of a fabricated F-P InAs/InP QD laser; (b) Schematic cross-sectional diagram of a shallow-etched ridge-waveguide InAs/InP QD laser

Fig. 2(a) shows the light output of the lasers as a function of drive current with blue dots for $11 \mathrm{GHz}$, red dashes for 25 $\mathrm{GHz}$, and green solid for $34.5 \mathrm{GHz}$ lasers at $20^{\circ} \mathrm{C}$. Fig. 2 (b) shows the lasing spectra at the indicated drive current for the three lasers. We have investigated optical linewidth for individually filtered longitudinal lasing modes by using an OE4000 automated laser phase noise measurement system and their timing jitters based on their RF beating signals. 

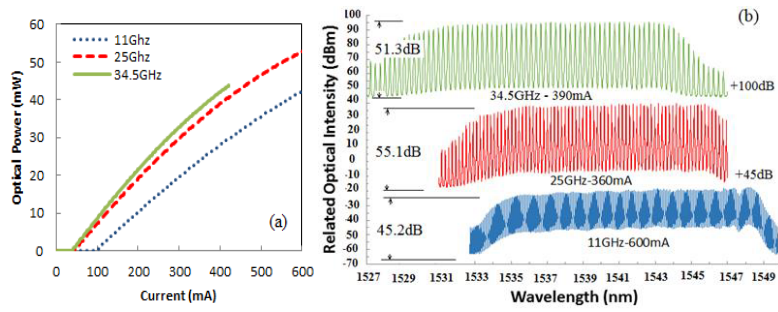

Fig. 2 (a) Light-current characteristics (b) Optical spectra of 11, 25 and 34.5 GHz QD MWLs at 0.01-nm resolution.

To demonstrate the performance of these lasers in a Tbit/s system we have used both PAM-4 and 16-QAM modulation schemes. Fig. 3(a) shows the experimental setup for PAM-4 and 16-QAM data transmission system where the optical modulation is performed by a dual polarization QAM transmitter. A PAM-4 signal is created using the arbitrary waveform generator (AWG) for a NRZ PRBS with a pattern length of $2^{15}-1$ bits at a symbol rate of 28 GBaud on two uncorrelated channels (IY and IX). Fig. 3(b) shows the Bit Error Rate (BER) as a function of received power for backto-back (B2B) and after $25 \mathrm{~km}$ of SMF transmission using a single filtered wavelength mode at $1547.855 \mathrm{~nm}$ (one of the 48 channels) in the $34.5 \mathrm{GHz}$ laser. The inset shows the measured eye diagram after $25 \mathrm{~km}$ SMF showing open eyes. The potential aggregate transmission capacity for the 48 channels available using the $34.5 \mathrm{GHz}$ laser is $5.376 \mathrm{Tbit} / \mathrm{s}$ (PAM-4 $48 \times 28$ GBaud PDM) over $25 \mathrm{~km}$ of a SMF transmission. Fig. 3(c) shows a 16-QAM constellation diagram at a symbol rate of 23 GBaud obtained from a filtered single laser mode at $1544.16 \mathrm{~nm}$ for the $25 \mathrm{GHz}$ laser. An error vector magnitude (EVM) of $7.74 \%$ and BER of $6.15 \times 10^{-6}$ were obtained. We obtained 16-QAM results at the base rate of 23 GBaud over 56 individual channels, which corresponds to an aggregate transmission capacity of $10.3 \mathrm{Tbit} / \mathrm{s}$ (16QAM 56x23 GBaud PDM) B2B.

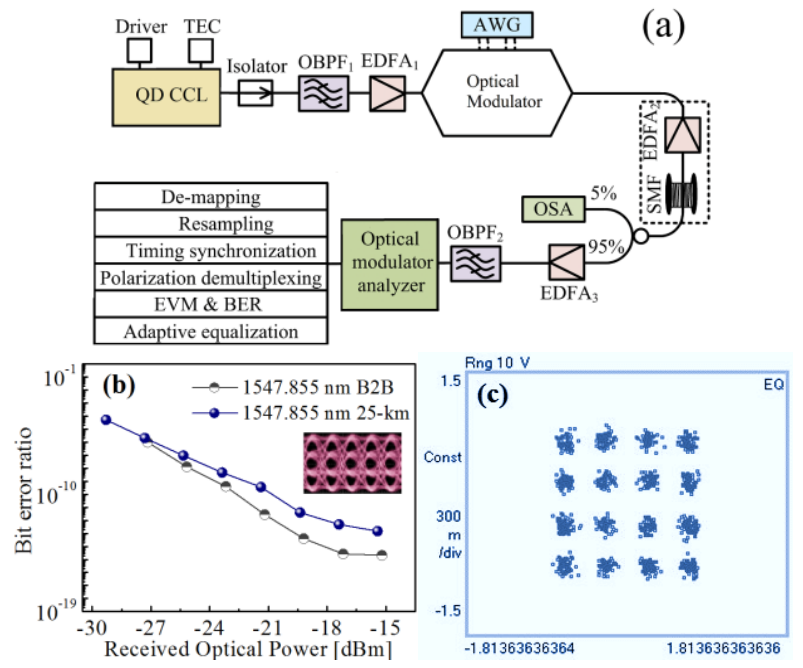

Fig. 3 (a) A schematic of PAM 4 and QAM data format system. (b) Measured BER versus received power for PAM 4 B2B and after $25-\mathrm{km}$ of SMF transmission using a comb line of $1547.855 \mathrm{~nm}$ of 34.5 GHz QD MWL. The inset shows corresponding eye diagram for $25-\mathrm{km}$ of SMF transmission. (c) A constellation eye diagram obtained from filtered channel of $1544.16 \mathrm{~nm}$ for the $25 \mathrm{GHz}$ QD MWL in the double polarization 16-QAM data format at 23 GBaud $\mathrm{B} 2 \mathrm{~B}$ transmission.

\section{QD DW DFB lasers for $5 G$ wireless networks}

The device was implemented in a $p$-n blocked buried heterostructure distributed feedback (BH DFB) laser with a novel synthesized aperiodic non-uniform diffraction grating layer placed below the InAs/InP QD active layer, which provides the distributed feedback such that two longitudinal modes lase simultaneously [7]. Fig. 4 shows its L-I curve, the insect shows its optical spectrum at $360 \mathrm{~mA}$ and their generated MMW signal of $46.8 \mathrm{GHz}$. The phase noise and RIN were measured experimentally for the two dominant optical modes. At $360 \mathrm{~mA}$, optical linewidths of $25.9 \mathrm{kHz}$ and $29.4 \mathrm{kHz}$ were measured for the two optical modes at $\lambda_{1}$ $=1539.522 \mathrm{~nm}$ and $\lambda_{2}=1539.896 \mathrm{~nm}$, respectively. Average RIN was measured as $-150.8 \mathrm{~dB} / \mathrm{Hz}$ for $\lambda_{1}$ and $-151.4 \mathrm{~dB} / \mathrm{Hz}$ for $\lambda_{2}$ over the frequency range from $10 \mathrm{MHz}$ to $20 \mathrm{GHz}$. For MMW over-fiber transmission link, $\lambda_{2}$ of the QD DWDFB laser was modulated using a $40 \mathrm{GHz}$ Mach-Zehnder intensity modulator (MZ-IM) with 1 Gbaud (2Gbps) PAM-4 signals generated through AWG and $\lambda_{1}$ was used as beat note signal for heterodyne MMW carrier generation. A clear eye diagram of PAM-4 signals of 1Gbaud transmission bandwidth along with transmitted/received signals were measured using Tektronix digital phosphor real-time oscilloscope. These results indicate the promising applications of the demonstrated QD DW-DFB laser as a compact mm-wave optical beat source in heterodyne $\mathrm{mm}$ wave communication systems of $5 \mathrm{G}$ and beyond.
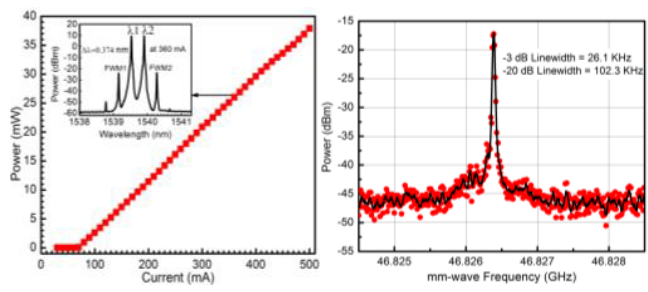

Fig. 4. L-I characteristics (inset shows the optical spectrum at 360 $\mathrm{mA}$ ) and their generated MMW signal.

\section{References}

1. Z.G. Lu "Quantum dot coherent comb lasers for terabit optical networking systems", (Invited paper) In Proc. Photonics West, San Francisco, CA, USA, Vol. 10921, 109210N (4 March 2019).

2. Z.G. Lu, et al. "312-fs pulse generation from a passive C-band InAs/InP quantum dot mode-locked laser," Opt. Express, 16, 10835-10840 (2008).

3. Z.G. Lu, et al. "An L-band monolithic InAs/InP quantum dot mode-locked laser with femtosecond pulses," Opt. Express, 17, 13609-13614 (2009).

4. Z.G. Lu, et al. "Ultra-high repetition rate InAs/InP quantum dot mode-locked lasers," Optics Communications, Vol. 284, No. 9, pp. 2323-2326 (May 2011).

5. Z.G. Lu, et al. "High performance InAs/InP quantum dot 34.462-GHz C-band coherent comb laser module," Opt. Express, Vol. 26, No. 2, 2160-2167(2018).

6. P. J. Poole, et al. "Growth of InAs/InP-based quantum dots for $1.55 \mu \mathrm{m}$ laser applications," J. Cryst. Growth 311(6), 1482-1486 (2009).

7. M. Rahim, et al. "Monolithic InAs/InP quantum dash dualwavelength DFB laser with ultra-low noise common cavity modes for millimeter-wave applications", Optics Express 27 (24), 35368-35375 (November 25, 2019). 